\title{
Rethinking research
}

Research and research publications in the recent past have shown an exponential increase in many ways and for several reasons. This increase is related to the availability of diverse sources of funding, monetary incentives, professional and academic awards and rewards for research, and enhanced opportunities for publication from increasing numbers of journals and conference proceedings published both in print and / or online. The SCImago Journal \& Country Rank, a publicly available portal that includes journals and country scientific indicators developed from the information contained in the Scopus ${ }^{\circledR}$ database (Elsevier B.V.) has ranked Sri Lanka 76 among 234 countries with 2379 citable documents and an H-index of 184 in 2019. The rank shows an improvement over 2014 when the rank was 84 and had 1298 citable documents.

The advances in information technology, ease of communication, and investment opportunities for research partnerships, coupled with curiosity and the thirst for high quality research have expanded the horizons of research leading to local and foreign collaborative research, inter and transdisciplinary research, and transfer of technology and biological material across borders. Given the relatively low government investment on research and development in Sri Lanka which remains consistently $<1 \%$ of GDP, collaborative research is of increasing importance as a source of funding and an opportunity to participate in and benefit from research, which otherwise may not be within the reach of Sri Lankan researchers. Researchers should keep in mind that intellectual property rights arising out of the processes and procedures, outcomes and benefits, commercial or otherwise that arise from such research have to benefit Sri Lanka.

A collaborative publication by the World Bank and Elsevier in 2019 has examined research and collaborations in South Asian countries focusing on research output citation impact, and report that intraregional, extra-regional and cross-sector research collaborations as important for these countries. South Asia's research output though modest, is growing rapidly with $1.7 \%$ rise in scholarly output between 2012 and 2016 even though outputs are reported as being at a low level relative to population size. Of the South Asian publications, a little less than $20 \%$ has been shown to be the product of international collaborations. Even though the citation impact of South Asian research is reported to be below the global average, the citation impact of research from Bangladesh, Sri Lanka, Nepal, Afghanistan, Bhutan and the Maldives exceeds the global average, which the report attributes to the boosting from highly impactful large scale research collaborations. Given the circumstances it is creditable that Sri Lanka published more papers per researcher than the world average.

Thus, research institutions, organisations, and funding agencies need to facilitate researchers in developing collaborations and fast track the process while conforming to the legal, administrative, and ethical requirements and ensuring the highest scientific standards. This is important for two reasons. First, the government has laid down a stringent process for approval of foreign collaborations, and rightly so. Secondly, should there be delays we stand to lose collaborative research opportunities, an important source of funds which has proved to give us high impact publications in relatively larger numbers.

At the same time there is a second area of research that needs to be addressed by all stakeholders, namely, the research waste. It is argued that research should not only advance the careers and reputations of researchers but should also bring benefit to people and society at large. Research findings need to feed into policy formulation, programme management, and be preferably linked to sustainable development. Implicit in doing research is an understanding that findings from research are utilised for the betterment of the society through evidence informed practice and policy formulation and funding agencies currently tend to explicitly seek information on these aspects. 
A publication in 2009 by Chalmers and Glasziou identify the sources of health research waste as: the research question not being relevant; design and methodology not being appropriate; the research has not led to full publication/s and even when published there are issues of not having access; and biased and unusable trial interventions reports. In 2017, the National Health Research Symposium in Sri Lanka attracted 504 abstracts of which $213(42.6 \%)$ had been rejected at review and re-review for reasons of poor quality $(35.6 \%)$ and due to ethics issues (7\%). Useful information on research waste, if there is any, will be explicitly clear if the organisers of research conferences and congresses, and research institutes and organisations published such information. In fact they could go one step further by exploring how many of published abstracts resulted in a full journal publication, had adequate merit to receive research awards and the findings had been forwarded to relevant authorities for consideration as evidence for policy formulation, or even only as feedback for appropriate action.
The third area is the role of research institutions and organisations in addressing the above two areas of concern as well as enhancing their own image in the research community. This can be achieved in many ways. Firstly, are means and methods by which research institutes and organisations support researchers to produce high quality research through provision of training, peer review, mentoring, funding and supporting publications, in place? To what extent do they have explicit published guidelines, instructions, codes of conduct, research governance strategies, and policies on addressing research misconduct and intellectual property rights?

I believe that the basic responsibilities of the research community are to, formulate the values and principles of research, define the criteria of appropriate behaviour in relation to research, achieve high quality standards, thoroughness and applicability of research, and prevent and respond to threats or violations of research integrity, and ensure the wellbeing of participants and citizens of Sri Lanka.

Rohini de Alwis Seneviratne 